\title{
A STUDY OF THE MECHANICS OF SPINAL INJURIES
}

\author{
Robert Roaf, OSWESTRy and LiVERPOOL, ENGLAND
}

The basic spinal unit consists of two intact vertebrae joined by an intervertebral disc, two posterior articulations and a number of ligaments (Fig. 1). There have been a number of studies of isolated intervertebral discs (Virgin 1951; Brown, Hansen and Yorra 1957) but there are few published accounts of the reactions of a complete spinal unit when subjected to a variety of forces. This paper describes the results of studies of such spinal units when subjected to forces of different magnitude and direction-compression, flexion, extension, lateral flexion, rotation and horizontal shear.

In particular I have been interested in the question of the discrepancy between the magnitude of the force and the resulting lesion. In other words, why, on one occasion, does a relatively small force produce a serious dislocation or major disc prolapse, whereas, on another occasion, a much larger force produces only a relatively minor vertebral crush? I believe that it is now possible to understand the underlying reasons for these differences and to reproduce all varieties of spinal injury experimentally by accurately controlled forces.

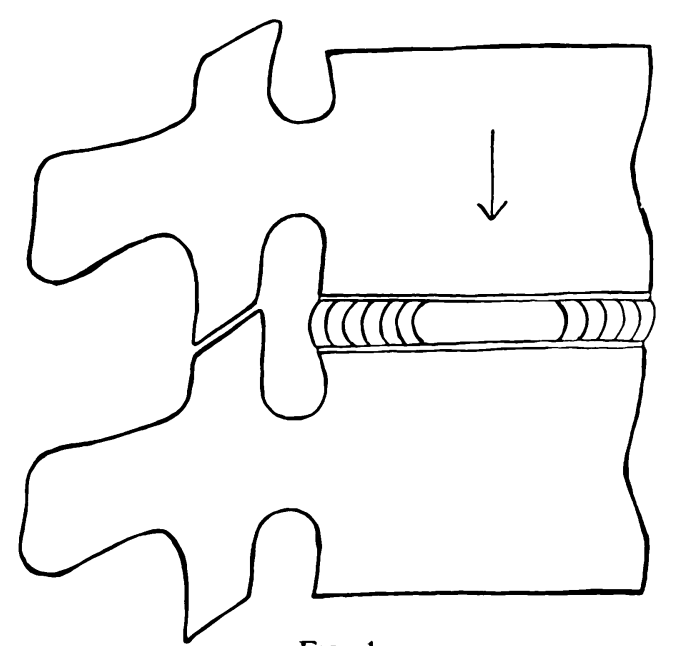

FiG. 1

A single spinal unit.

Definitions-A force has direction and magnitude. A given tissue-bone or ligament-breaks when sufficient force is applied in the right direction to a localised area for a given time. The meaning of compression force is obvious; distraction is nearly as clear, though it is a pity that the more exact term extension has been wrongly applied to backward flexion. By flexion and extension I mean rotation in the sagittal plane about the normal axis of rotation; thus, in flexion there is relaxation of the anterior ligaments and tightening of the posterior ligaments. Hyperflexion means disruption of the posterior elements by a distracting force in the sagittal plane. Extension and hyperextension are the opposite. Rotation force is a couple applied in the horizontal plane; in practice rotation and horizontal shear forces are usually combined.

\section{MATERIAL AND METHODS}

Portions of healthy cervical, thoracic and lumbar spines were obtained, mostly from children and young adults, as soon after death as possible. The cause of death was either injury or acute non-spinal infection. In a few cases specimens from older patients were used, 


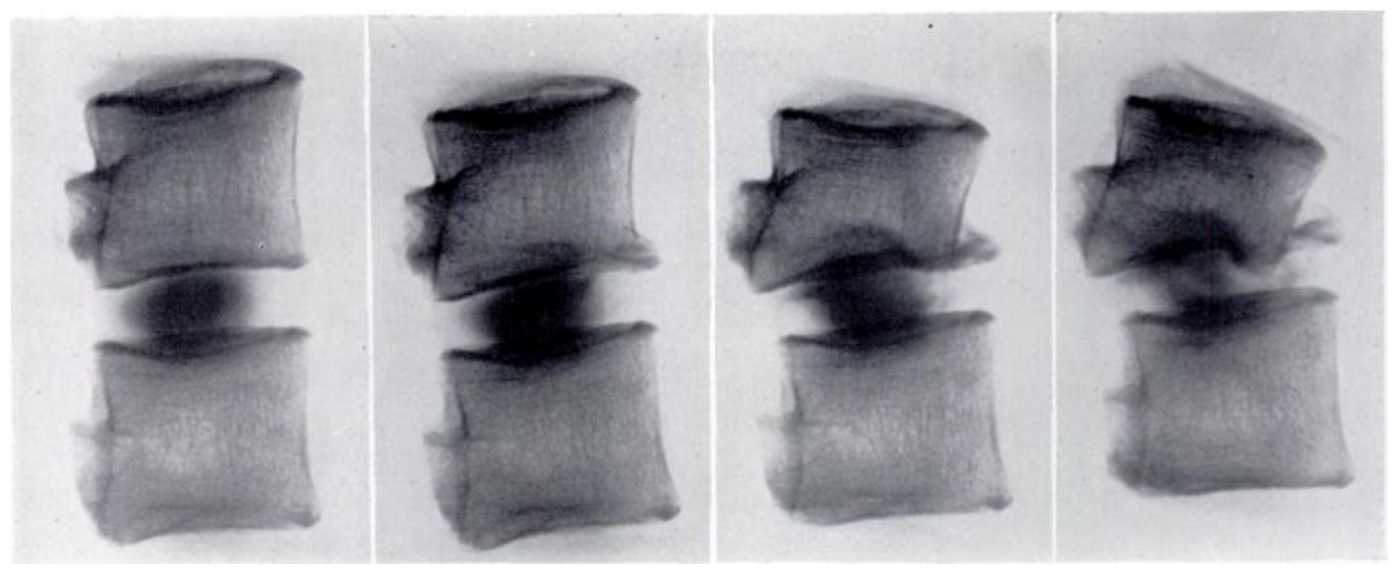

FIG. 2

Normal discographs under increasing pressure.

either to investigate the effect of compression in spinal units in which the nucleus pulposus had become fibrotic, or to investigate the effect of rotation forces upon an arthritic cervical spine. The specimens were at once placed in a " deep-freeze." Before use they were immersed in warm water for twelve to twenty-four hours. For certain experiments the nucleus pulposus was injected with a 20 per cent solution of potassium iodide made to suitable viscosity with glycerine and sufficient methylene blue to produce the colour, thus enabling the nucleus to be studied both radiographically (Fig. 2) and visually. This injection did not appear to alter the mechanical properties of the disc; in other words there was no observed difference between injected and uninjected specimens.

For ease of analysis the forces were usually applied slowly; for example, compression forces were applied with a standard Denniston testing machine. It is realised that most human injuries are the consequence of rapidly acting forces, but on the few occasions when a rapidly acting force was used the general pattern of response was similar to the pattern when slowly acting forces were used.

\section{RESULTS}

COMPRESSION FORCES

Intact disc and vertebrae-Provided the nucleus pulposus retains its normal turgor (Petter 1933), there is a uniform pattern of response seen most characteristically in the lumbar region where the nuclei pulposi are relatively larger and their reactions can be studied more easily.

As vertical pressure increases there is a very slight bulge of the annulus but no alteration in the shape of the nucleus pulposus, and the major distortion is a bulge of the vertebral end-plate. Indeed it is only as this happens that the two vertebrae move closer to each other (diminution of disc space); the annular bulge is secondary to this and is not due to pressure from the nucleus pulposus. (The annular bulge is more marked when there is no nucleus pulposus, just as an under-inflated pneumatic tyre bulges more than a well-inflated one.)

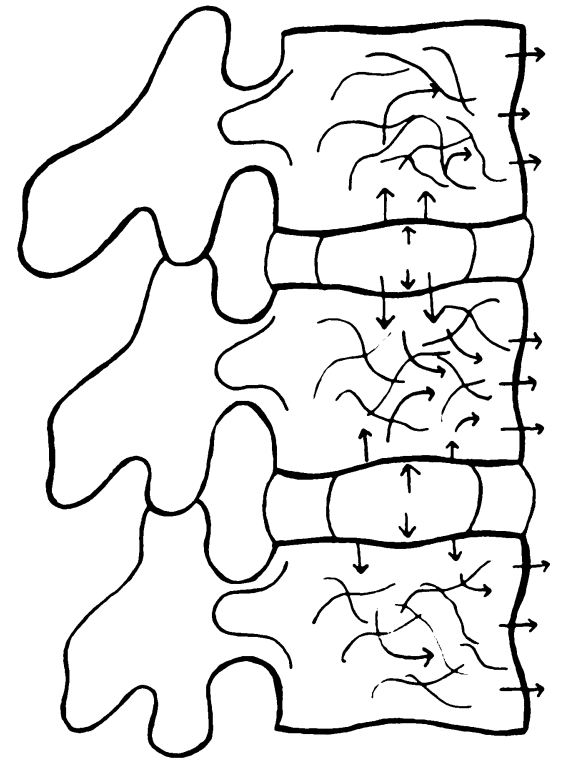

Fig. 3

Mechanism of spinal shock absorption. Arrows show direction of pressure gradients and fluid movements. 
Bulging of the end-plate causes blood to be squeezed out of the cancellous bone of the vertebral body. This is the first sign of increasing compression and is accompanied by a fall in pressure (Fig. 3). It is considered that this is an important shock-absorbing mechanism in the spine. As pressure increases the end-plate bulges more and finally cracks (Figs. 4 to 6 ).

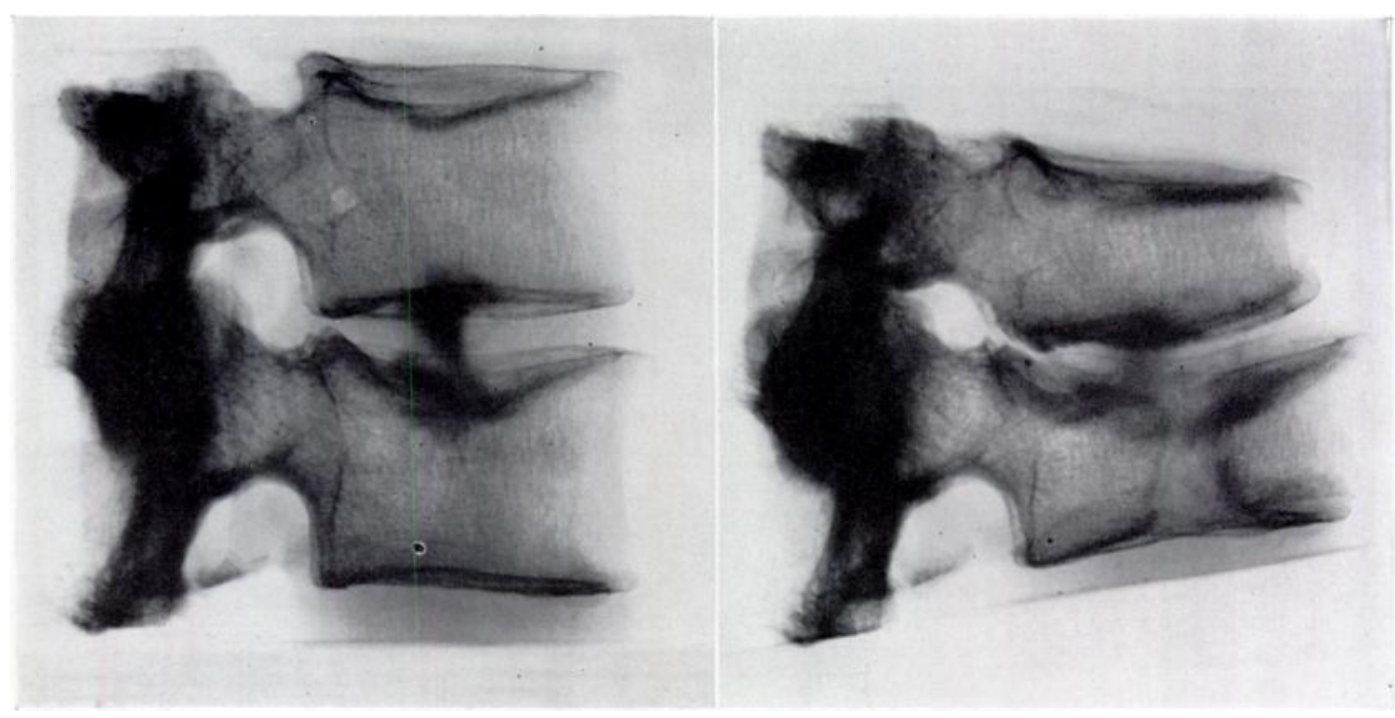

FIG. 4

FIG. 5

Figure 4-Bulging of end-plate. Figure 5-Vertical split in body. Nucleus pulposus undeformed.

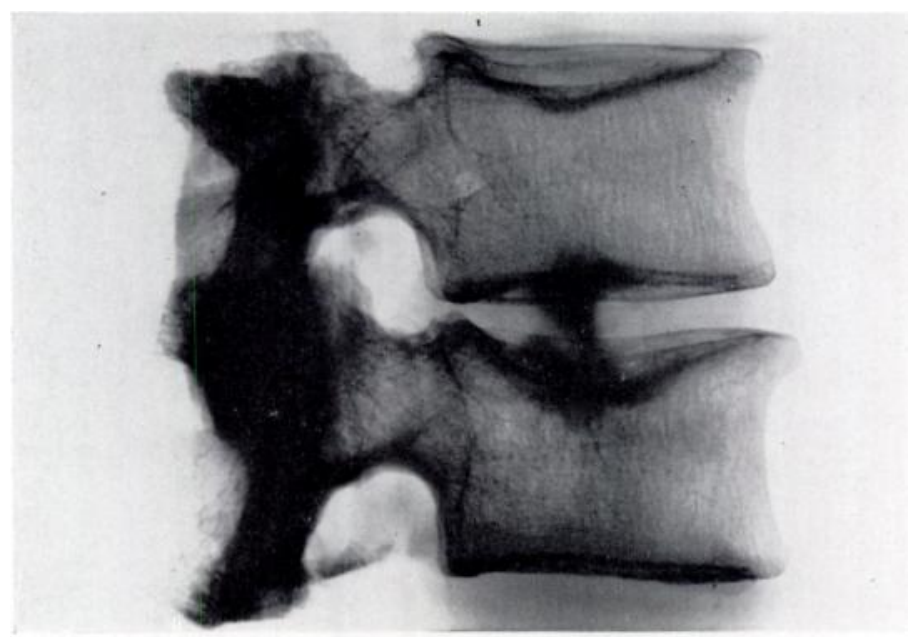

Fig. 6

End-plate fracture. Nucleus undeformed.

Up to this point there has been relatively little bulging of the annulus. Once the end-plate has been broken, nuclear material is displaced into the vertebral body (Figs. 7 and 8). The disc has now lost its turgor and the annulus begins to bulge like the walls of a flat tyre. Continued pressure leads to disintegration of the vertebral body. If the line of force is oblique the line of fracture is oblique (Fig. 9).

Figure 10 shows typical distance-pressure graphs for complete spinal units and for isolated wet and dry vertebrae. The results have been uniform in eighteen experiments: 1) the 
intact disc is more resistant to vertical compression than the vertebral body; 2) on compression of an intact spinal unit the vertebral end-plate bulges; 3 ) this causes oozing of blood from the vertebral body, which appears to be a major shock-absorbing mechanism (Fig. 3); 4) there is little, if any, movement or distortion of the normal nucleus pulposus.

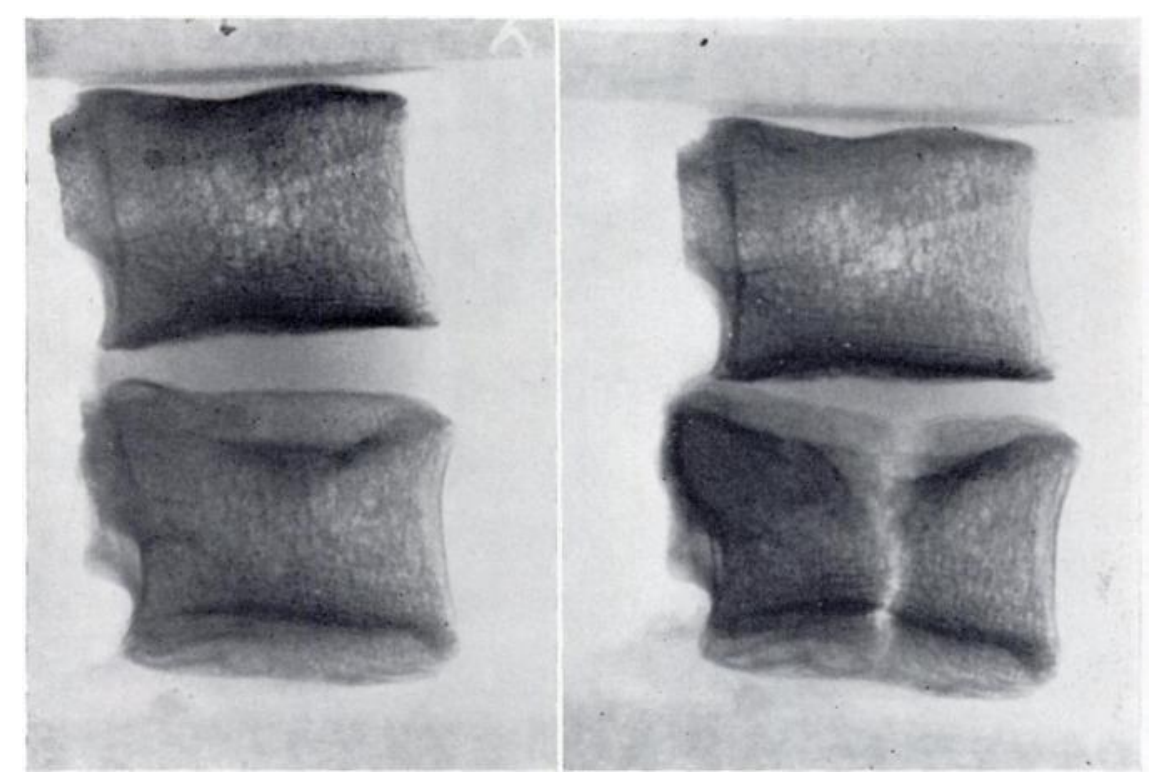

Fig. 7

End-plate fracture leading to vertical fracture of body.

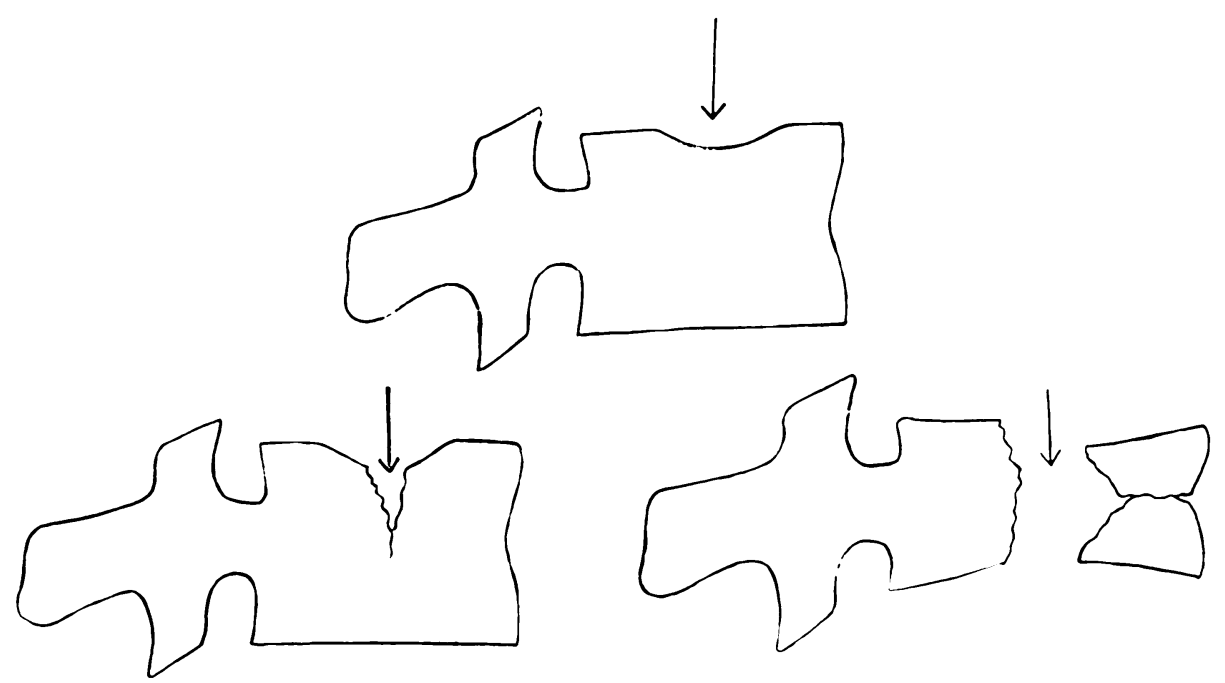

FIG. 8

Diagrams of sequence of events.

There is no significant bulging of the annulus until the nucleus has lost its turgor in consequence of nuclear displacement into the vertebral body from collapse of the vertebral end-plate.

There was never any evidence of nuclear material pressing upon the annulus and causing a localised protrusion, as has been often suggested (Armstrong 1952). Indeed, if the annulus 


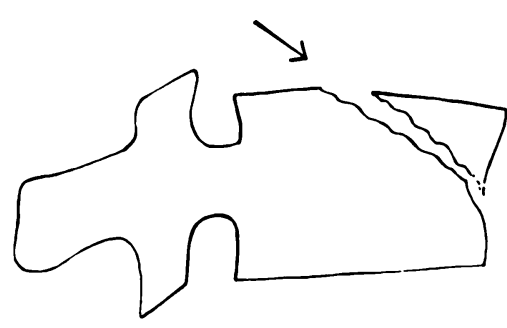

Fig. 9

Oblique fracture of vertebral body when compression and forward shear forces are combined.

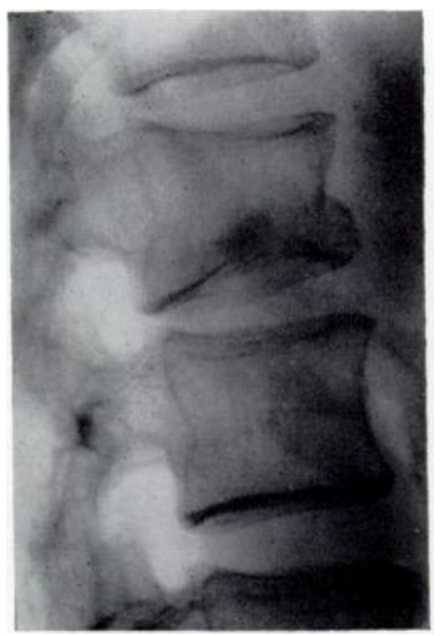

is artificially weakened at a given point, nuclear fluid exudes and, being fluid, is dispersed evenly over the surface. There is no annular bulge until the whole disc disintegrates, and the site of the bulge is not determined by the site of annular weakness and nuclear seepage. Disc protrusions, as observed clinically, consist of annular material. Indeed how could freely flowing fluid cause a localised nerve root compression?

Compression of specimens from older subjects in which the nucleus was no longer fluid, and asymmetrical compression, produced a different form of fracture. Pressure under these conditions is mainly transmitted through the annulus and the result is either tearing of the annulus, general collapse of the vertebrae due to buckling of the sides (Fig. 11) or a marginal plateau fracture (Figs. 12 and 13).

Compression of denucleated spinal units-The healthy nucleus pulposus of a child or young adult in life is a viscous liquid very similar to the contents of a ganglion. Its shape is maintained by the surrounding walls of the cavity in which it lies-vertebral end-plate and annulus fibrosus. The mechanical properties of the intervertebral disc depend on a normal nuclear

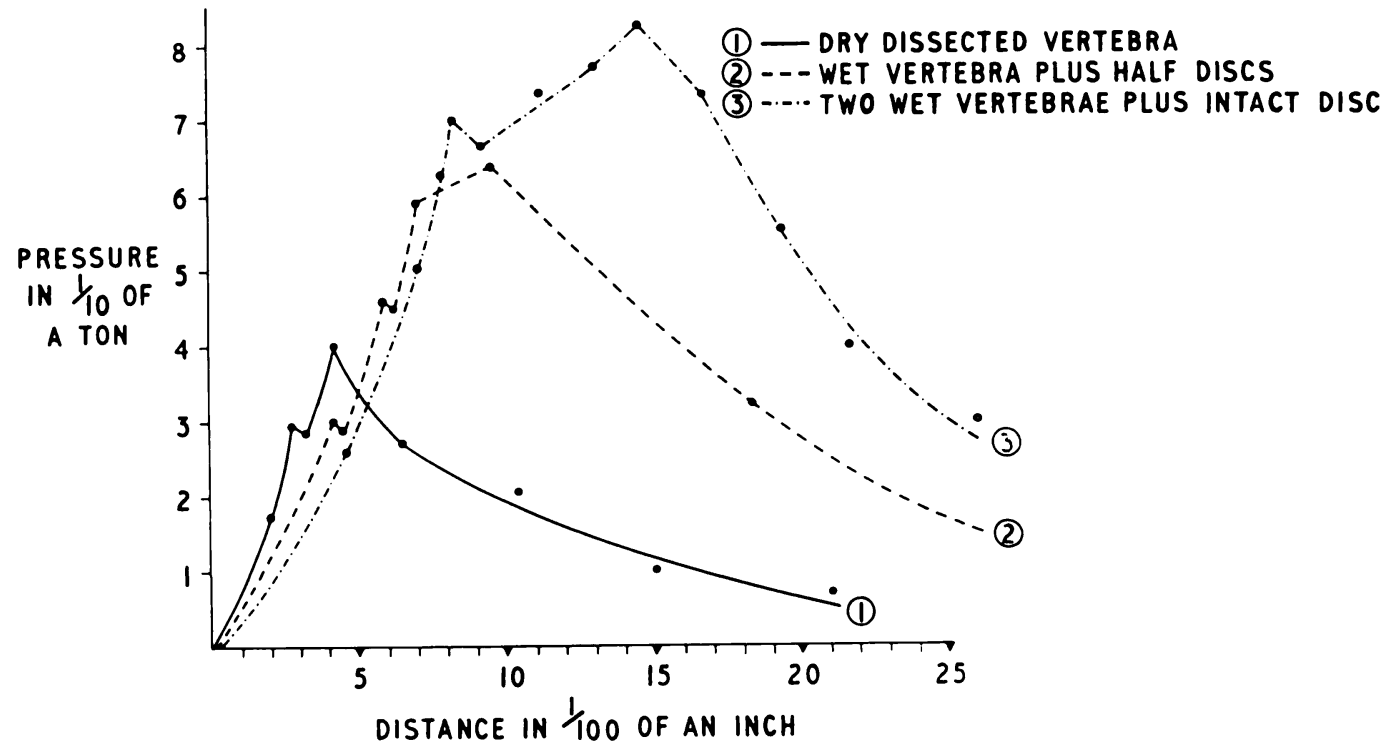

FIG. 10

Distance-pressure graphs for a complete spinal unit, a wet and a dry vertebra. 
A STUDY OF THE MECHANICS OF SPINAL INJURIES


FIG, 11

General collapse of the vertebral body.
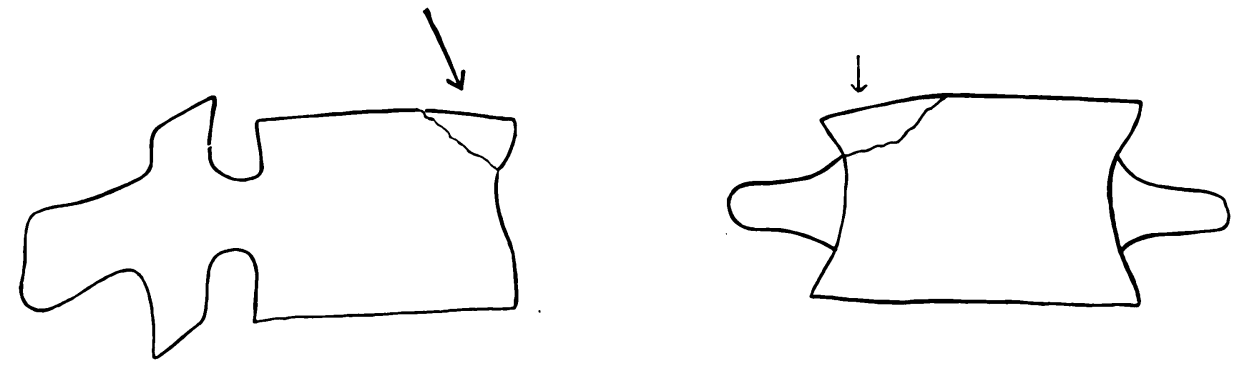

FIG. 12

Marginal plateau fracture (not to be confused with the slice fracture due to rotation).
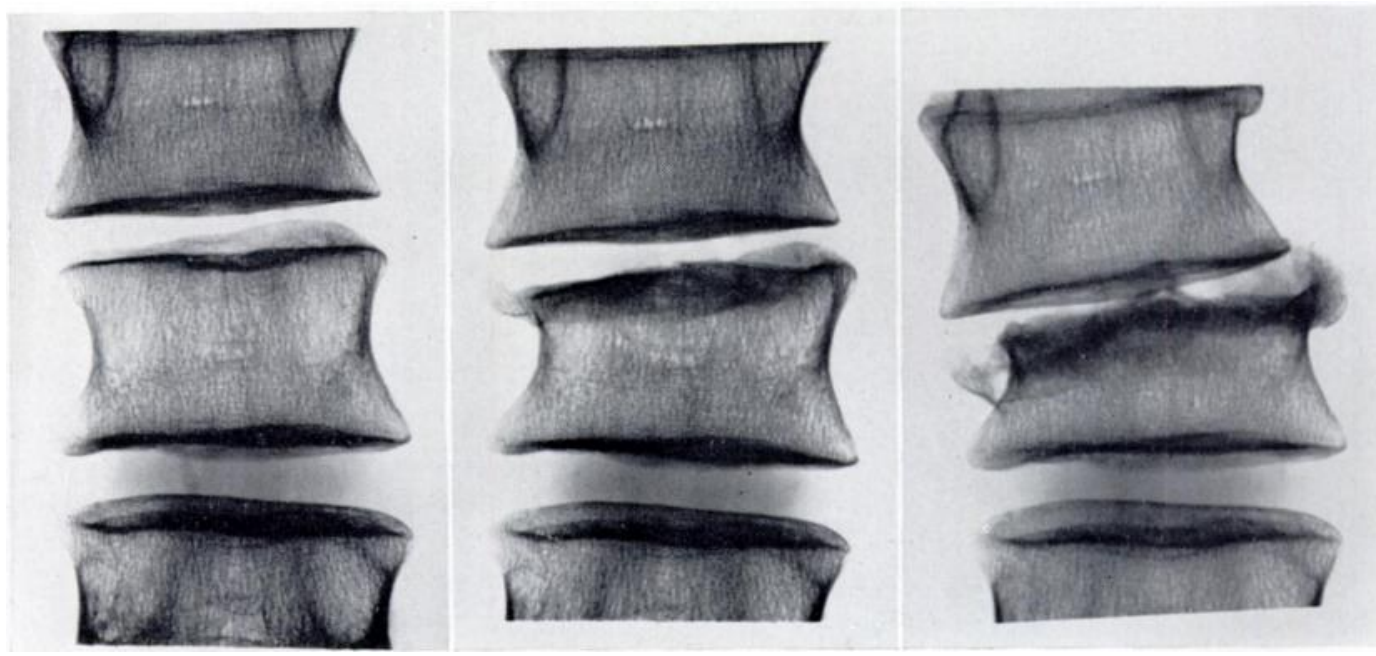

FIG. 13

Marginal plateau fracture.

VOL. 42 B, NO. 4, NOVEMBER 1960

$\mathrm{K}$ 


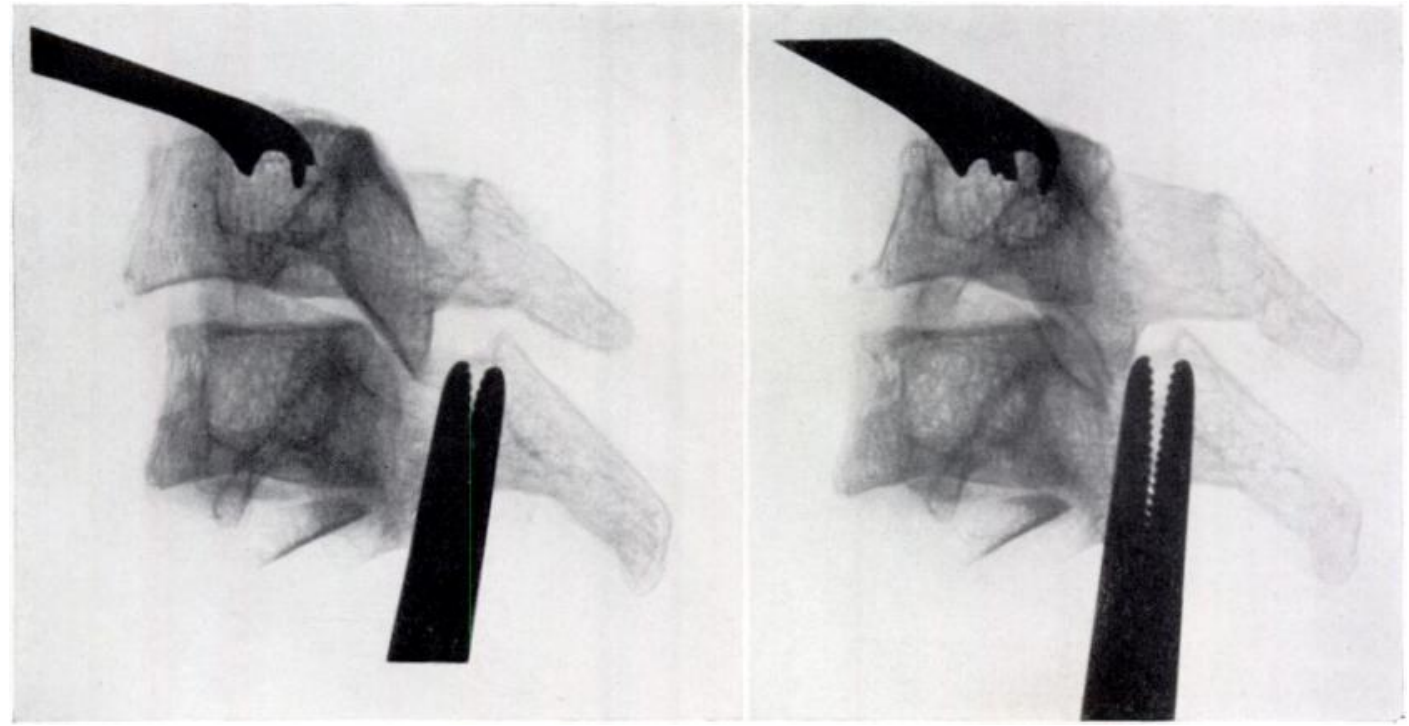

Fig. 14

Abnormal mobility after enucleation.

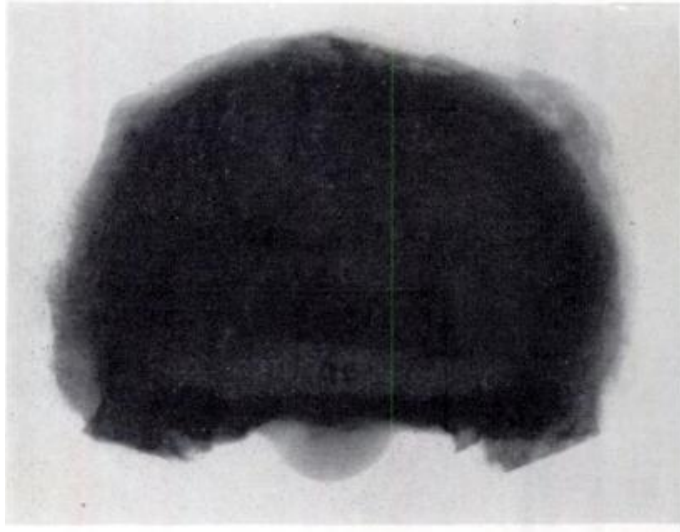

FIG. 15

Typical posterior disc (annulus) protrusion caused by gentle compression after denucleation by anterior root.

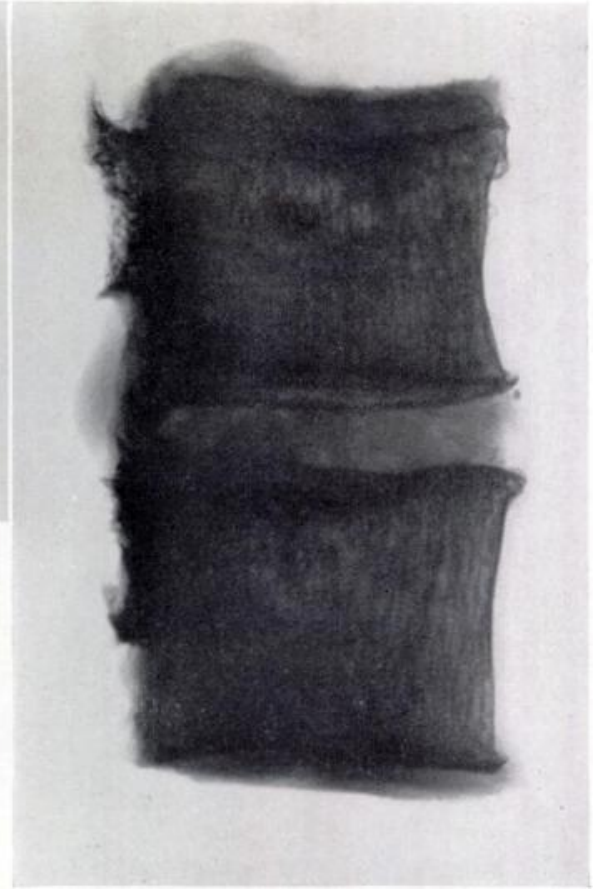

turgor. The disc can be compared to a plant cell. If the nucleus is normally hydrated it has turgor and retains its shape. If the nucleus is dehydrated (mechanically or otherwise) it collapses and loses its normal mechanical properties. If intranuclear pressure increases the evidence is that the vertebral end-plate bulges: this may be an important cause of back pain.

After mechanical removal of the fluid nucleus pulposus there is abnormal mobility between the vertebral bodies (Roaf 1958) (Fig. 14). If such a "denucleated" unit is compressed the result is quite different. The annulus at once bulges, there is no bulging of the vertebral end-plate and no oozing of blood from the vertebral body. If the compression is continued, typical disc (annular) prolapses, as seen at operation, are produced (Figs. 15 and 16). 
The vertebral end-plate fracture has considerable medico-legal importance. A patient may easily sustain a compression injury without a radiographically visible fracture. Nevertheless, he may have sustained a vertebral end-plate fracture with intracorporeal nuclear protrusion and loss of disc turgor. His disc is now vulnerable, and a short time later a relatively minor injury may cause a severe and incapacitating annular prolapse for which the first, not the second, injury was the real cause.

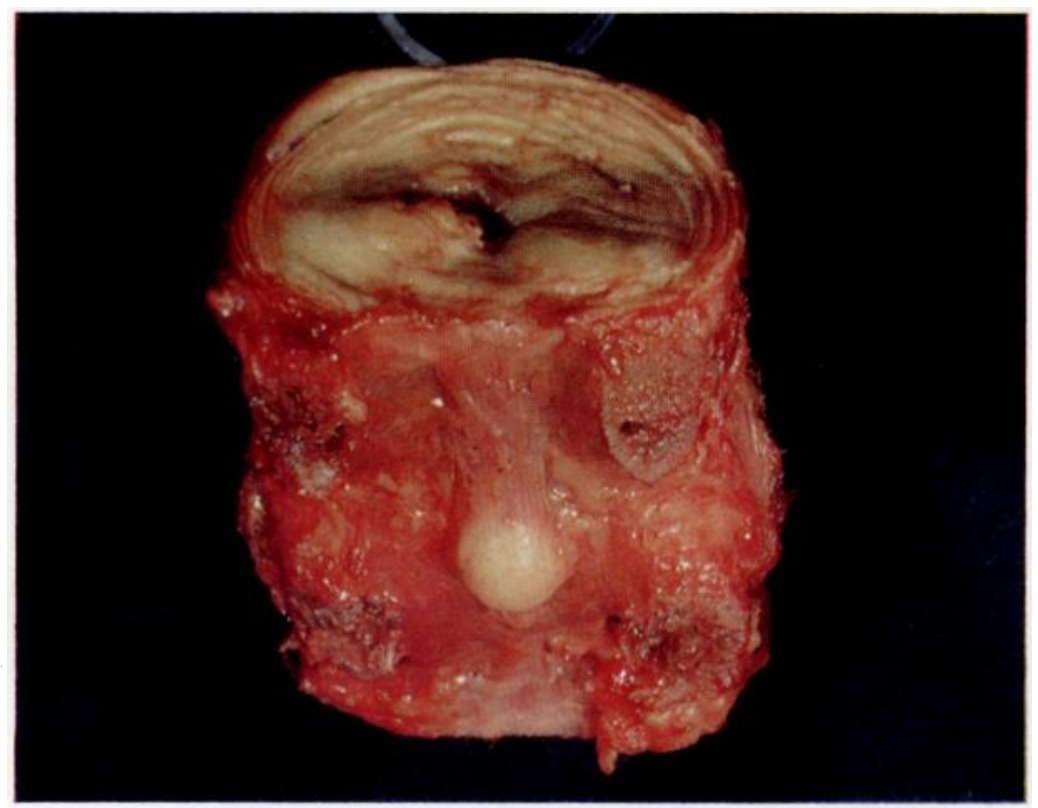

Fig. 16

Photograph of specimen the radiographs of which are shown in Figure 15.

FLEXION FORCES

I have never succeeded in producing pure hyperflexion injuries of a normal intact spinal unit. Before the posterior ligaments rupture, the vertebral body always becomes crushed. This is what one would expect from an elementary calculation of moments. Pressure at the fulcrum must be three to four times as great as tension on the posterior ligaments, whose breaking point is about $400 \mathrm{lb}$. (Brown et al. 1957). Pressure on the nucleus is therefore $1,200-1,600 \mathrm{lb}$., which exceeds the breaking point of the vertebral end-plate.

There was never any significant alteration in either the position or the shape of the healthy nucleus pulposus (Figs. 17 to 22) or any significant annular bulge. If any bulge occurs it is always posteriorly on extension and anteriorly on flexion. Similarly, after denucleation annular bulging occurs anteriorly on forward flexion, not posteriorly as is often suggested.

\section{EXTENSION AND LATERAL FLEXION}

Very similar results were obtained with extension and lateral flexion (Figs. 17 to 22). It was found impossible to rupture the anterior longitudinal ligament of the cervical spine by a pure hyperextension force: crush fracture of the neural arch occurred first. Here again there was no detectable alteration in shape or position of the nucleus pulposus and no significant annular bulge posteriorly on extension. After denucleation there was excessive mobility and an annular bulge posteriorly on extension.

\section{ROTATION}

The intervertebral discs, joints and ligaments were found to be very resistant to compression, distraction, flexion and extension, but very vulnerable to rotation and horizontal 
shearing forces. In the cervical region particularly, manual rotation will produce tearing of ligaments and dislocation. If the spine is held in slight flexion and a rotation force is applied the posterior ligaments, joint capsules and posterior longitudinal ligament tear in that order, and a typical dislocation results (Figs. 23 and 24). This may or may not be associated with a

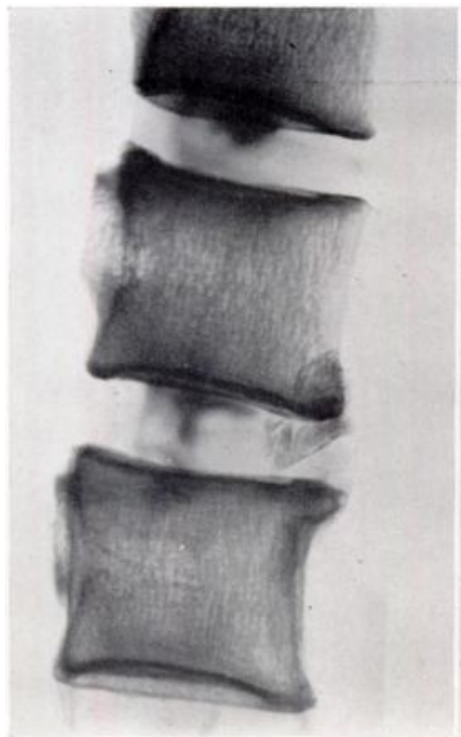

FIG. 17

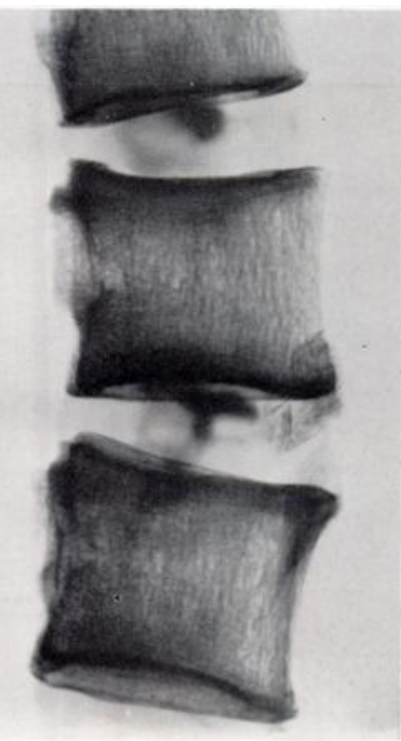

FIG. 18

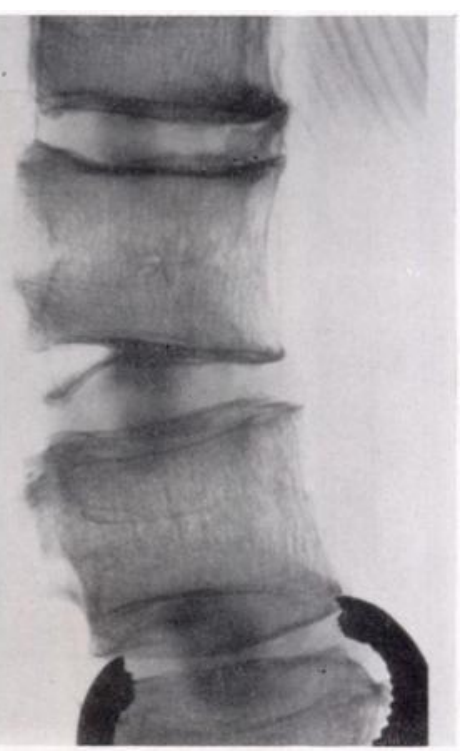

FIG. 19



FIG. 20



FIG. 21

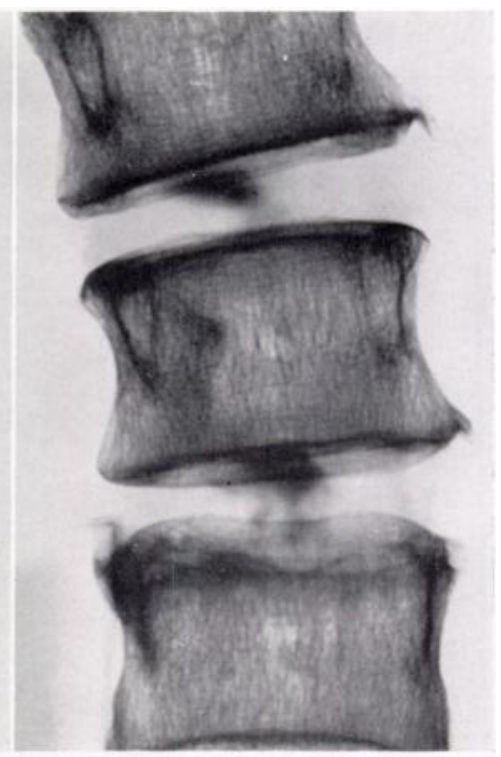

FIG. 22

Nucleographs of spine in hyperflexion and hyperextension. The neural arches have been removed to increase the range of movement. Even so, there is hardly any change in shape or position of the nucleus pulposus. Figure 17-Flexion. Figure 18-Extension. Figure 19-Hyperflexion. Note avulsion of end-plate. Figure 20Extension. Figures 21 and 22-Lateral flexion.

fracture of the vertebral body depending on the amount of associated compression force. It is clear that the common clinical appearances of a cervical dislocation or fracture-dislocation usually attributed to flexion or hyperflexion are really due to rotation (Figs. 23 and 24). Similarly, it has not been possible to rupture the anterior longitudinal ligament by 


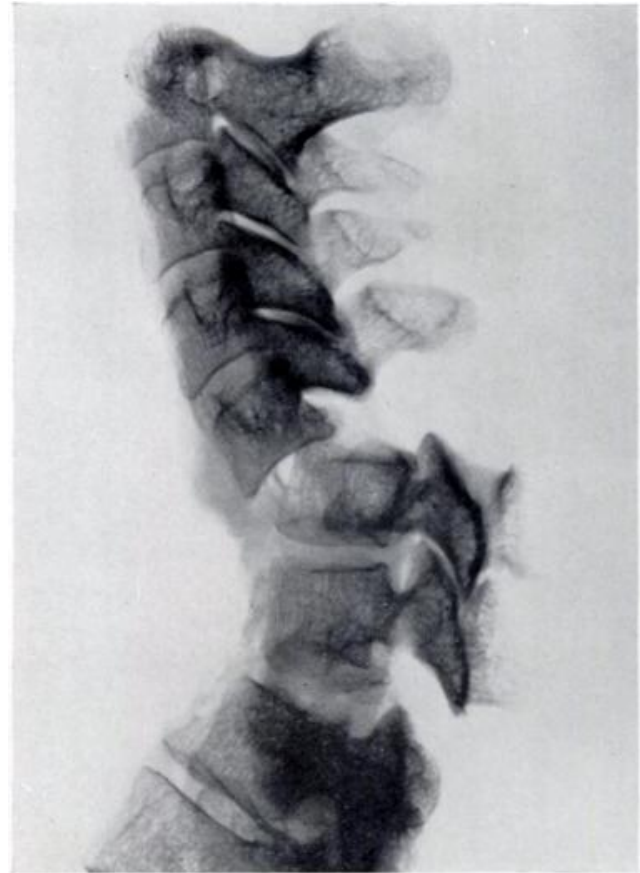

FIG. 23



FIG. 24

Figure 23-Dislocation produced by gentle rotation force. Figure 24-The same specimen after reduction.

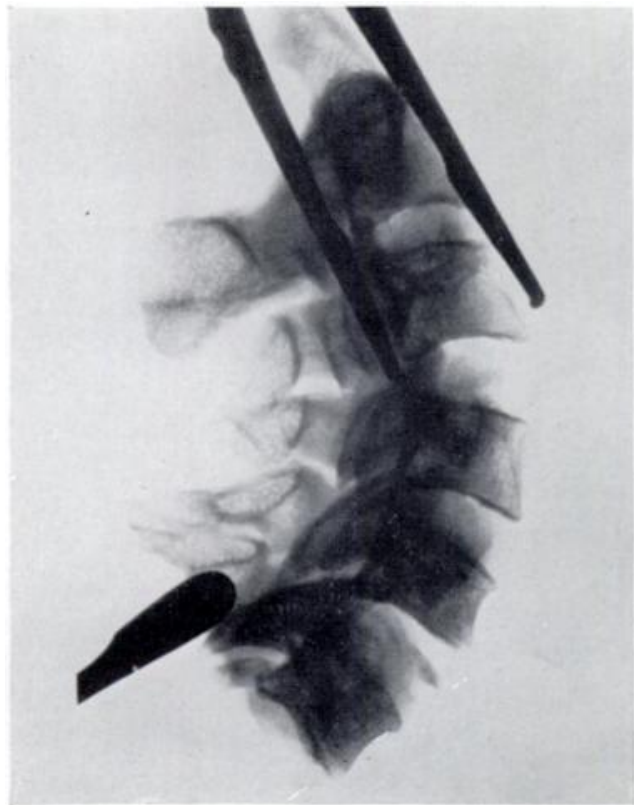

Fig. 25



Fig. 26

Figure 25-Rupture of anterior longitudinal ligament produced by rotation force. Figure 26-The same specimen after reduction.

VOL. 42 B, NO. 4, NOVEMBER 1960 


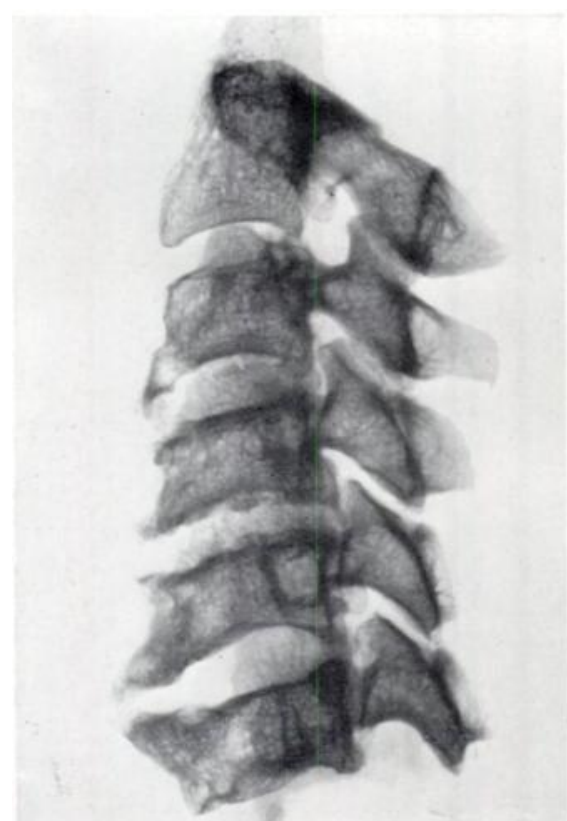

FIG. 27

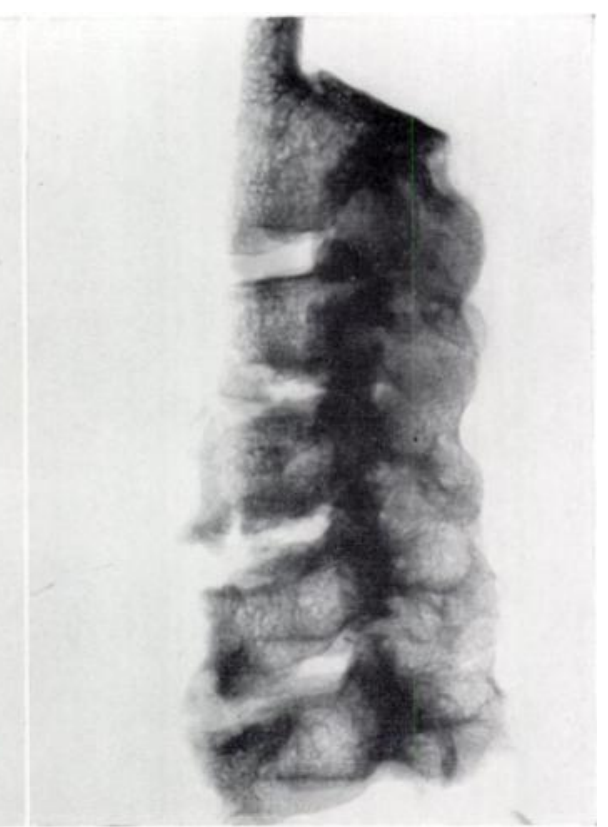

FIG. 28

Rotation force applied to arthritic spine. Figure 27-Lateral view: no fracture seen. Figure 28-Oblique view, showing marked displacement with vertical fracture of the vertebral body. Anterior longitudinal ligament appears intact

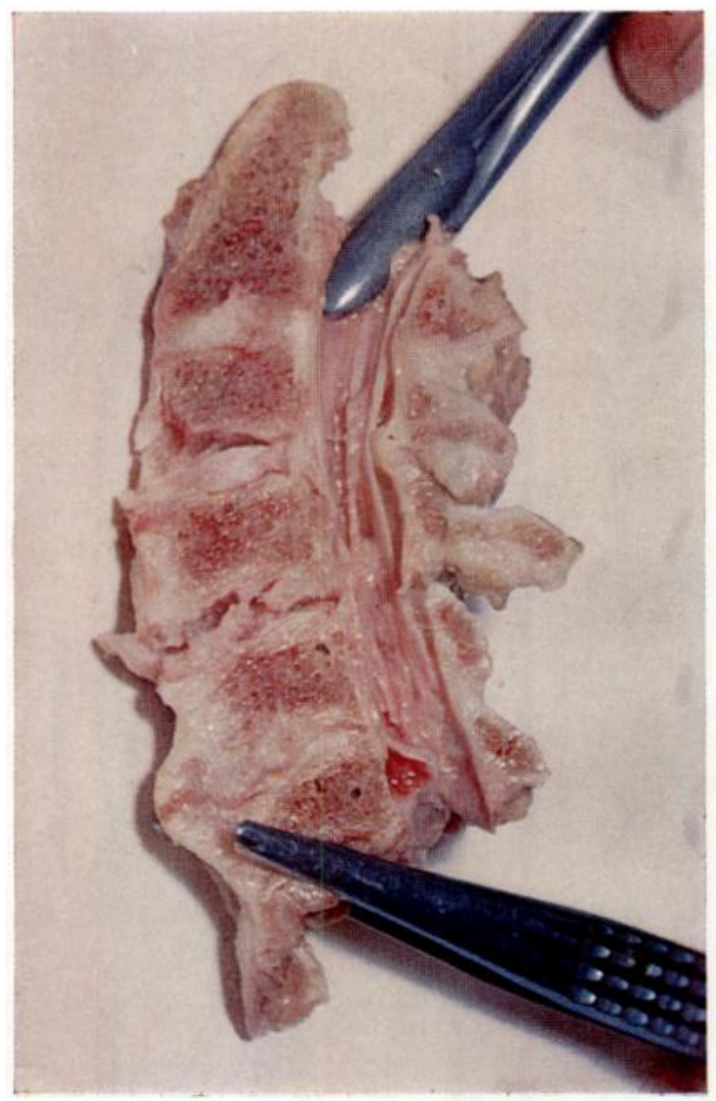

Fig. 29

Photograph of specimen after rotation injury. 


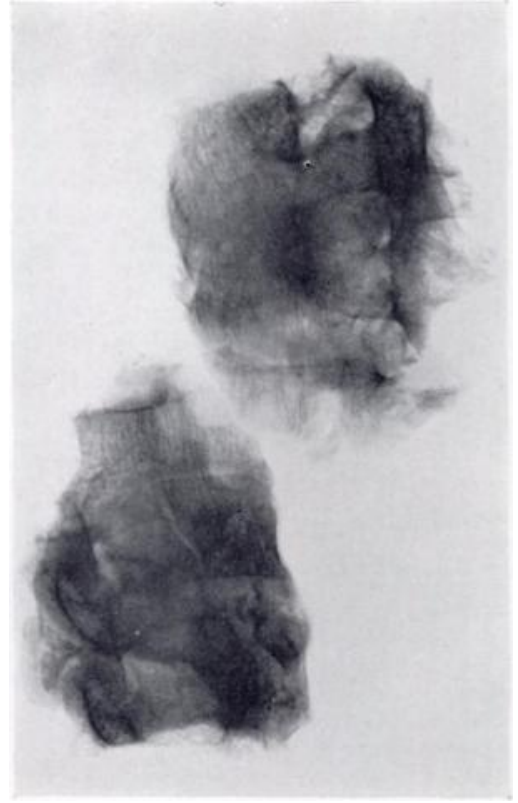

FIG. 30

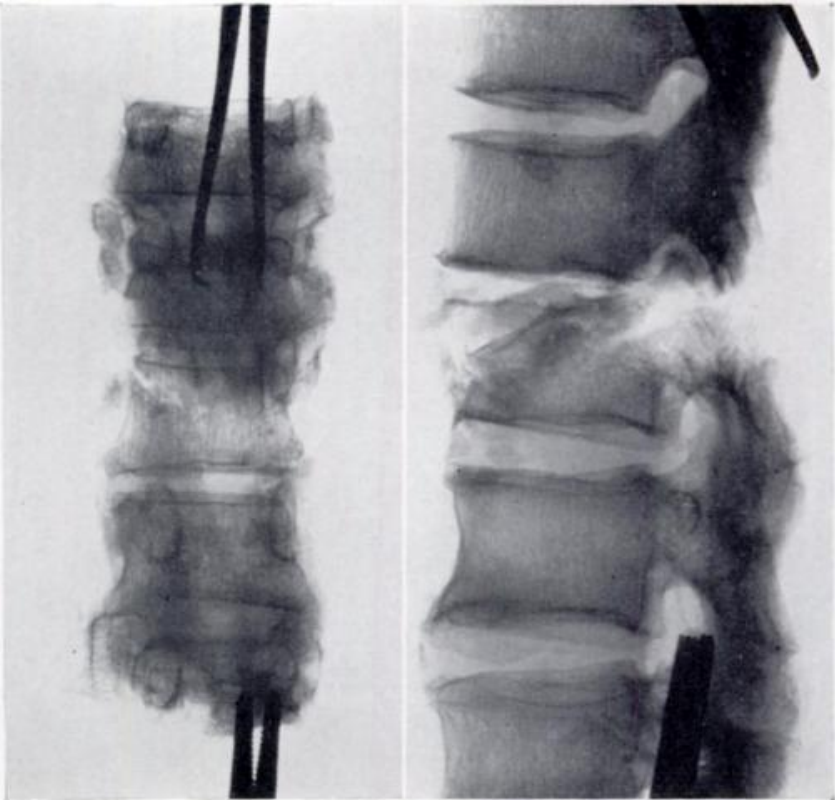

Fig. 31

Figure 30-Rotation injury of thoraco-lumbar region. Figure 31-The same specimen, closed.

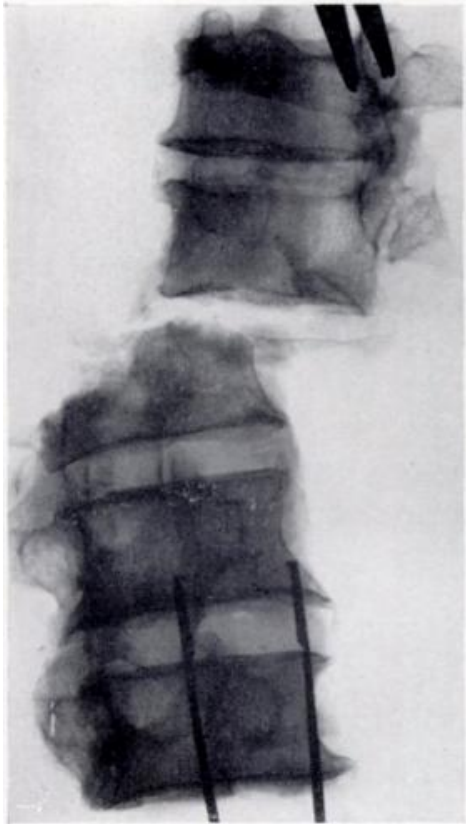

FiG. 32

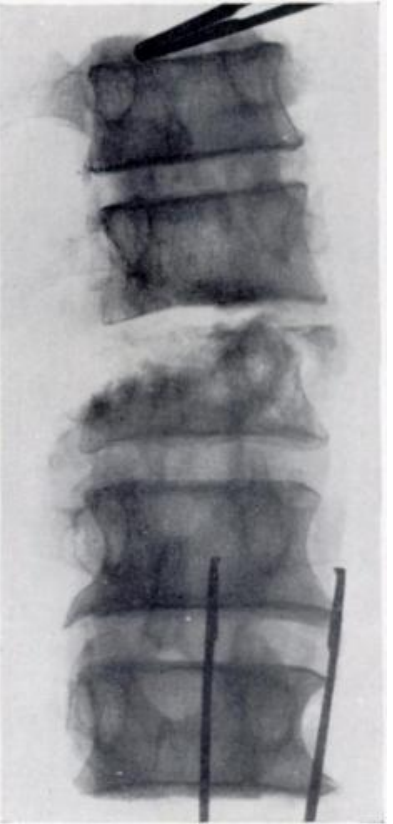

Fig. 33

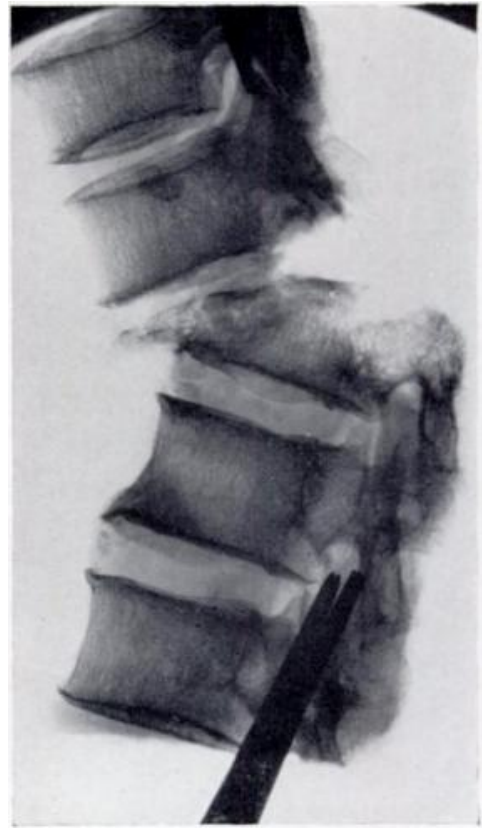

FIG. 34

Figure 32-Rotation injury, open. Figure 33-Same specimen, closed. Figure 34-Another rotation injury.

VOL. 42 B, NO. 4, NOVEMBER 1960 
hyperextension; the neural arches always fracture first. If the spine is rotated in the extended position the anterior longitudinal ligament easily ruptures (Figs. 25 and 26). The so-called hyperextension injury is really a rotation injury. If the nucleus pulposus has lost its turgor these effects are produced more easily, and rotation and horizontal shear forces will produce considerable intervertebral displacement or subluxation even without rupture of the anterior longitudinal ligament (Figs. 14, 27 to 29). Similarly, in the thoraco-lumbar region a relatively small rotation force will produce a dislocation (Figs. 30 to 36 ). If the spine is held in flexion

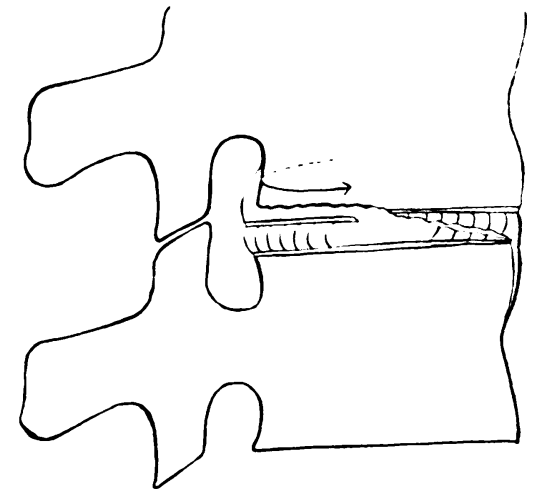

Fig. 35

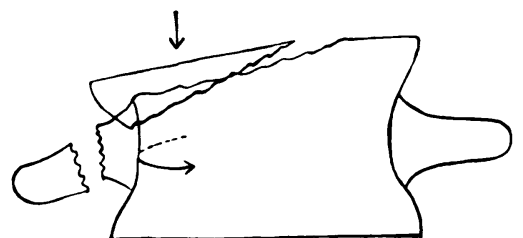

FIG. 36

FIGS. 35 AND 36

Figure 35-Diagram of pure rotation injury. Figure 36-Diagram of rotation combined with slight compression. This injury is unstable and must be differentiated from the stable marginal plateau fracture. (Compare Figure 12.)

this can occur without fracture of the articular processes, although it is more common to have an associated articular process fracture. Holdsworth and Hardy (1953) have pointed out that spontaneous reduction may occur and that radiological examination alone may fail to reveal the extent of the damage and the degree of spinal instability (Figs. 30 to 36). My clinical and experimental findings confirm their views on this point.

\section{SUMMARY}

1. Compression forces are mainly absorbed by the vertebral body. The nucleus pulposus, being liquid, is incompressible. The tense annulus bulges very little. On compression the vertebral end-plate bulges and blood is forced out of the cancellous bone of the vertebral body into the perivertebral sinuses. This appears to be the normal energy-dissipating mechanism on compression.

2. The normal disc is very resistant to compression. The nucleus pulposus does not alter in shape or position on compression or flexion. It plays no active part in producing a disc prolapse. On compression the vertebral body always breaks before the normal disc gives way. The vertebral end-plate bulges and then breaks, leading to a vertical fracture. If the nucleus pulposus has lost its turgor there is abnormal mobility between the vertebral bodies. On very gentle compression or flexion movement the annulus protrudes on the concave aspect-not on the convex side as has been supposed.

3. Disc prolapse consists primarily of annulus; it occurs only if the nucleus pulposus has lost its turgor. It then occurs very easily as the annulus now bulges like a flat tyre.

4. I have never succeeded in producing rupture of normal spinal ligaments by hyperextension or hyperflexion. Before rupture occurs the bone sustains a compression fracture. On the other hand horizontal shear, and particularly rotation forces, can easily cause ligamentous rupture and dislocation.

5. A combination of rotation and compression can produce almost every variety of spinal injury. In the cervical region subluxation with spontaneous reduction can be easily produced by rotation. If disc turgor is impaired this may occur with an intact anterior longitudinal 
ligament and explains those cases of tetraplegia without radiological changes or a torn anterior longitudinal ligament. The anterior longitudinal ligament can easily be ruptured by a rotation force and in my experience the so-called hyperextension and hyperflexion injuries are really rotation injuries.

6. Hyperflexion of the cervical spine or upper thoracic spine is an anatomical impossibility. In all spinal dislocations a body fracture may or may not occur with the dislocation, depending upon the degree of associated compression. In general, rotation forces produce dislocations, whereas compression forces produce fractures.

I would like to express my thanks to Mr W. G. Davies, Superintendent Radiographer at the Robert Jones and Agnes Hunt Orthopaedic Hospital, for invaluable assistance in these experiments.

\section{REFERENCES}

Armstrong, J. R. (1952): Lumbar Disc Lesions. Edinburgh and London: E. \& S. Livingstone Ltd.

Brown, T., Hansen, R. J., and Yorra, A. J. (1957): Some Mechanical Tests on the Lumbosacral Spine with Particular Reference to the Intervertebral Discs. Journal of Bone and Joint Surgery, 39-A, 1,135.

Holdsworth, F. W., and Hardy, A. (1953): Early Treatment of Paraplegia from Fractures of the Thoracolumbar Spine. Journal of Bone and Joint Surgery, 35-B, 540.

Petter, C. K. (1933): Methods of Measuring the Pressure of the Intervertebral Disc. Journal of Bone and Joint Surgery, 15, 365.

Roaf, R. (1958): The Treatment of Resistant Scoliosis. Proceedings of the Royal Society of Medicine (Section of Orthopaedics), 51, 237.

VIrgin, W. J. (1951): Experimental Investigations into the Physical Properties of the Intervertebral Disc. Journal of Bone and Joint Surgery, 33-B, 607. 